\title{
LA FERTILIZACIÓN MINERAL COMO COMPLEMENTO A LA FERTILIZACIÓN CON ABONO ORGÁNICO EN EL CULTIVO DEL CAFÉ
}

\author{
Fernando Farfán Valencia (D) * José Enrique Baute Balcázar (ID **
}

\begin{abstract}
Farfán-Valencia, F., \& Baute-Balcázar, J. E. (2020). La fertilización mineral como complemento a la fertilización con abono orgánico en el cultivo del café. Revista Cenicafé, 71(1), 48-53. https://doi. org/10.38141/10778/1119
\end{abstract}

Con el propósito de evaluar diferentes fuentes potásicas minerales naturales, en mezcla con pulpa de café descompuesta para emplearlas como fertilizantes en el cultivo del café, en la Estación Experimental de Pueblo Bello, ubicada en el municipio de Pueblo Bello, departamento de Cesar, zona cafetera norte de Colombia; se evaluaron dos fuentes de potasio: Sulfato de Potasio $\left(\mathrm{K}_{2} \mathrm{SO}_{4}\right)$ y Sulpomag $\left(\mathrm{K}_{2} \mathrm{SO}_{4} .2 \mathrm{MgSO}_{4}\right)$, sobre la producción en kilogramos.ha ${ }^{-1}$ de café pergamino seco; esta producción fue comparada con la obtenida al fertilizar con fertilizantes inorgánicos, con materia orgánica y un testigo sin fertilizar; el café fue establecido a densidades de 3.922, 6.060 y 7.843 plantas/ha en un sistema agroforestal. De los resultados se obtuvo que en la fertilización del café bajo sombrío pueden adoptarse alternativas como la aplicación de materia orgánica en mezcla con fuentes de potasio como el $\mathrm{K}_{2} \mathrm{SO}_{4} .2 \mathrm{MgSO}_{4}$, comercializado como Sulpomag, siempre que se establezcan altas densidades de siembra. El café establecido a densidades inferiores a 6.000 plantas por ha, puede ser fertilizado con fuentes orgánicas o inorgánicas.

Palabras clave: Sistemas agroforestales, café con sombrío, fertilización orgánica.

\section{MINERAL FERTILIZATION AS A COMPLEMENT TO ORGANIC FERTILIZATION IN COFFEE CULTIVATION}

\begin{abstract}
With the purpose of evaluating different natural mineral potassium sources mixed with decomposed coffee pulp to use them as fertilizers in coffee crops, two potassium sources were evaluated: Potassium Sulfate $\left(\mathrm{K}_{2} \mathrm{SO}_{4}\right)$ and Sulpomag $\left(\mathrm{K}_{2} \mathrm{SO}_{4} \cdot 2 \mathrm{MgSO}_{4}\right)$ on the production in kilograms.ha ${ }^{-1}$ of dry parchment coffee. The study was done at the Pueblo Bello Experimental Station, located in the municipality of Pueblo Bello, department of Cesar, northern Colombian coffee region. This production was compared with the one obtained with inorganic fertilizers, with organic matter and an unfertilized control; coffee was established at densities of 3.922, 6.060 and 7.843 plants.ha $^{-1}$ in an agroforestry system. The results showed that the fertilization of coffee with shade allow to provide alternatives such as the application of organic matter mixed with potassium sources such as $\mathrm{K}_{2} \mathrm{SO}_{4} \cdot 2 \mathrm{MgSO}_{4}$, commercialized as Sulpomag, provided that high planting densities are established. Coffee established at densities of less than 6.000 plants per ha can be fertilized with organic or inorganic sources.
\end{abstract}

Keywords: Agroforestry systems, shade coffee, organic fertilization.

\footnotetext{
* Investigador Científico II. Disciplina de Fitotecnia, Centro Nacional de Investigaciones de Café, Cenicafé. Manizales, Caldas, Colombia. https://orcid.org/0000-0003-0976-8828.

** Asistente de Investigación. Disciplina de Experimentación, Centro Nacional de Investigaciones de Café, Cenicafé. Manizales, Caldas, Colombia. https://orcid.org/0000-0001-5540-7746.
} 
La producción de café en Colombia tuvo un crecimiento cercano al $90 \%$ en los últimos siete años, al pasar de 7,7 millones en 2012 a 14,7 millones de sacos de 60 kilos de café verde en 2019 (Federación Nacional de Cafeteros de Colombia [FNC], 2020). Teóricamente para haber alcanzado este volumen productivo, en las fincas cafeteras se produjeron cerca de 2.130.000 toneladas de pulpa de café fresca (Montilla et al., 2008), de las cuales se obtendrían alrededor de 948.459 toneladas de abono orgánico a los seis meses de su transformación (Roa et al., 1999). Si se aplicaran $3,0 \mathrm{~kg}$ de este material orgánico por planta de café y por año, sería suficiente para fertilizar cerca de 52.692 hectáreas de café, con una densidad promedio de 6.000 plantas/ha. Lo que contribuiría a estimular el uso de los subproductos de la producción cafetera y a hacer empleo racional de los fertilizantes inorgánicos; teniendo en cuenta que la aplicación podría afectar los costos de producción, por incremento de la mano de obra para la fertilización con pulpa de café descompuesta.

De otro lado, para su mejor expresión en cuanto a producción, el café requiere de suelos con altos contenidos de materia orgánica $(>8,0 \%)$, ricos en nutrientes especialmente potasio y suelos con $\mathrm{pH}$ entre 5,0 y 5,5; pero se cultiva café en regiones con suelos donde, por su material parental entre otros, son evidentes condiciones contrarias a las anteriormente descritas, por ejemplo suelos de la Unidad Malabar, derivados de cenizas volcánicas, ácidos, con bajos contenidos de materia orgánica y de potasio, comunes en los municipios cafeteros de Convención, Arboleda, Teorama, San Calixto en Norte de Santander, algunos municipios como González y Río de Oro en el departamento de Cesar, o municipios como Calarcá, Armenia y La Tebaida, en el departamento del Quindío, en los cuales se encuentran los suelos de la Unidad Quindío, derivados de cenizas volcánicas (González, 2013).

Bajo estas condiciones de cultivo es fundamental implementar las prácticas necesarias de adecuación del suelo, tendientes a nivelar los tenores de $\mathrm{pH}$, incrementar los contenidos de materia orgánica y nutrientes, especialmente potasio, con el propósito de cubrir las expectativas socio-económicas del caficultor. Cuzato et al. (2014) sostienen que el rendimiento del café aumenta con el suministro de potasio, independiente de la fuente. Las prácticas de adecuación deben establecerse desde la siembra y continuar con ellas durante todos los ciclos productivos del cultivo, tendientes a mantener la producción alta y estable. Sadeghian (2008) plantea que entre las alternativas para corregir la acidez del suelo es necesaria la aplicación de cales y el uso de abonos orgánicos, aspecto en el cual los subproductos del beneficio del café, como la pulpa descompuesta, podrían hacer un gran aporte.

En la Estación Experimental de Pueblo Bello de Cenicafé, ubicada en el departamento de Cesar, se realizó una investigación tendiente a evaluar las mezclas de abono a base de pulpa de café y fuentes de potasio, para fertilizar el café establecido a diferentes densidades de siembra, y obtener el máximo rendimiento.

\section{MATERIALES Y MÉTODOS}

Sitio de estudio. La investigación se realizó en la Estación Experimental de Pueblo Bello, ubicada en el municipio de Pueblo Bello, departamento de Cesar. En la Tabla 1 se presentan las características de clima y suelos de la localidad.

Material vegetal. Se utilizó café Variedad Castillo $^{\circledR}$, establecido bajo un sombrío de Inga 
edulis (guamo santafereño), con densidades cercanas a los 70 árboles/ha.

Tratamientos. Se evaluaron 15 tratamientos producto de la combinación de dos factores; tres densidades de siembra del café y cinco alternativas de fertilización, como se describen en la Tabla 2.

Diseño experimental. Los tratamientos fueron asignados aleatoriamente, de acuerdo con el diseño experimental bloques completos al azar, en arreglo de parcelas divididas, donde el factor de bloqueo fue el gradiente de fertilidad del lote, la parcela principal fueron las densidades de siembra del café y las subparcelas las alternativas de fertilización.

Características de las parcelas. En la Tabla 3 se presentan las características de las parcelas experimentales. El campo experimental ocupó un área total cercana a 2,2 ha.

Análisis estadístico: Análisis de varianza al $5 \%$ bajo el diseño experimental propuesto, con la variable de respuesta y se aplicó la prueba de comparación de Tukey al 5\%.

Tabla 1. Características de clima y suelos, Estación Experimental Pueblo Bello, Pueblo Bello-Cesar.

\begin{tabular}{|c|c|c|c|}
\hline \multicolumn{2}{|c|}{ Localización geográfica } & \multicolumn{2}{|c|}{ Características de suelos } \\
\hline Latitud & $10^{\circ} 25^{\prime}$ & $\mathrm{pH}$ & 4,6 \\
\hline Longitud & $73^{\circ} 34^{\prime}$ & Materia orgánica (\%) & 7,1 \\
\hline Altitud (m) & 1.100 & Nitrógeno (\%) & 0,3 \\
\hline \multicolumn{2}{|c|}{ Características climáticas } & Fósforo (mg.kg-1) & 9,5 \\
\hline Temperatura media $\left({ }^{\circ} \mathrm{C}\right)$ & 21,2 & Potasio $\left(\mathrm{cmol}_{(+)} \cdot \mathrm{kg}^{-1}\right)$ & 0,5 \\
\hline Precipitación (mm) & 2.050 & Calcio $\left(\mathrm{cmol}_{(+)} \cdot \mathrm{kg}^{-1}\right)$ & 3,4 \\
\hline Brillo solar (horas año) & 2.380 & Magnesio $\left(\mathrm{cmol}_{(+)} \cdot \mathrm{kg}^{-1}\right)$ & 1,0 \\
\hline Humedad relativa $(\%)$ & 74,0 & Ecotopo & $402 \mathrm{~A}$ \\
\hline
\end{tabular}

Tabla 2. Densidades de siembra por alternativas de fertilización del café. Estación Experimental Pueblo Bello, Pueblo Bello-Cesar.

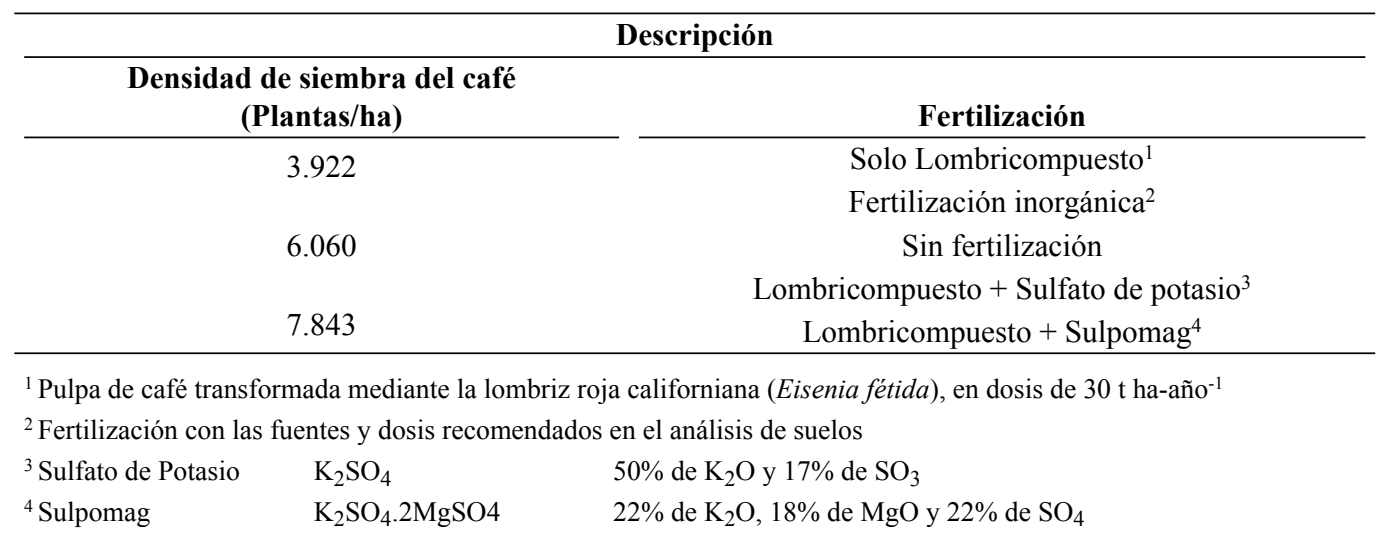




\section{RESULTADOS Y DISCUSIÓN}

Producción. En la Tabla 4 se presenta la producción acumulada de cinco cosechas (2010-2014) de café pergamino seco c.p.s. $\left(\mathrm{kg} \mathrm{ha}^{-1}\right)$, en cafetales establecidos en tres densidades de siembra.

El análisis de varianza para el modelo experimental propuesto no mostró significancia estadística $(p>0,05)$ para la interacción densidad de siembra del café $x$ fertilización, lo que indica un efecto independiente de los factores sobre la producción. Por lo tanto se realizaron pruebas de comparación de medias, Tukey $(5,0 \%)$, de cada densidad de siembra evaluada, registrando bajo la densidad de siembra del café de 3.900 plantas/ha, que no hay diferencias estadísticas entre las fuentes de fertilización estudiadas; puede inferirse que a bajas densidades de siembra y bajo sombrío es indiferente la fuente de fertilizante aplicado al cultivo, con una producción promedio bajo este número de plantas por hectárea de $4.554 \mathrm{~kg} \mathrm{ha}^{-1}$ de café pergamino, acumulados en cinco cosechas. Jessy (2011) encontró respuesta a la aplicación de fertilizantes al café bajo sombrío después del quinto año, con menor estrés por deficiencia hídrica en el suelo en plantas en las cuales su fertilización estuvo suplementada con fuentes de potasio.

Bajo la densidad de siembra del café a 6.000 plantas/ha la más baja producción, $4.051 \mathrm{~kg} \mathrm{ha}^{-1}$

Tabla 3. Número de plantas de café y de sombrío por parcela y parcela efectiva.

\begin{tabular}{|c|c|c|c|c|}
\hline \multirow{2}{*}{$\begin{array}{l}\text { Área parcela } \\
\text { Efectiva }\left(\mathbf{m}^{2}\right)\end{array}$} & \multirow{2}{*}{$\begin{array}{c}\text { Distancias de siembra } \\
\text { del café }(\mathbf{m})\end{array}$} & \multirow{2}{*}{$\begin{array}{c}\text { Distancias de siembra } \\
\text { del sombrío }(\mathrm{m})\end{array}$} & \multicolumn{2}{|c|}{ Plantas efectivas } \\
\hline & & & Café & Sombrío \\
\hline 364,7 & $1,5 \times 1,70$ & $12 \times 12$ & 143 & 1 \\
\hline 364,7 & $1,5 \times 1,10$ & $12 \times 12$ & 221 & 1 \\
\hline 364,7 & $1,5 \times 0,85$ & $12 \times 12$ & 286 & 1 \\
\hline
\end{tabular}

Tabla 4. Producción acumulada de cinco cosechas $\left(\mathrm{kg} \mathrm{ha}^{-1}\right.$ de c.p.s.) y tres densidades de siembra. Estación Experimental Pueblo Bello, Pueblo Bello-Cesar.

\begin{tabular}{|c|c|c|c|}
\hline \multirow[b]{2}{*}{ Fertilización } & \multicolumn{3}{|c|}{ Densidad de siembra del café (plantas/ha) } \\
\hline & 3.922 & 6.060 & 7.843 \\
\hline 1 & $4.566 \mathrm{a}$ & $5.197 \mathrm{a}$ & $6.136 \mathrm{~b}$ \\
\hline 2 & $4.630 \mathrm{a}$ & $5.269 \mathrm{a}$ & $5.305 \mathrm{c}$ \\
\hline 3 & $4.422 \mathrm{a}$ & $4.051 \mathrm{~b}$ & $4.515 \mathrm{~d}$ \\
\hline 4 & $4.473 \mathrm{a}$ & $5.084 \mathrm{a}$ & $6.206 \mathrm{~b}$ \\
\hline 5 & $4.680 \mathrm{a}$ & $5.194 \mathrm{a}$ & $7.014 \mathrm{a}$ \\
\hline c.v & 0,40 & 0,38 & 0,23 \\
\hline
\end{tabular}

Fertilización: (1) Solo lombricompuesto; (2) Fertilización inorgánica; (3) Sin fertilización; (4) Lombricompuesto + Sulfato de potasio; (5) Lombricompuesto + Sulpomag. Letras distintas representan diferencia estadística entre tratamientos según la prueba de Tukey al 5\%. 
de café pergamino seco, acumulados durante cinco cosechas, se registró con el tratamiento testigo (sin fertilización). En general, no se registraron diferencias estadísticas al comparar las producciones obtenidas al fertilizar el café con las diferentes fuentes evaluadas, con un promedio de la producción de 5.186 $\mathrm{kg} \mathrm{ha}{ }^{-1}$ de café pergamino seco. Ochoa et al. (2000) sostienen que la fertilización con materia orgánica debe ser suplementada con diversas fuentes entre las que se destacan las fosfóricas, para obtener los mejores índices de crecimiento y rendimiento, de lo contrario las plantas de café manifestarán limitadas producciones acumuladas.

Al incrementar la densidad de siembra del café a 7.800 plantas/ha se registraron diferencias estadísticas, según prueba de comparación Tukey $(5,0 \%)$, entre la producción al fertilizar el café con materia orgánica complementada con sulpomag y la fertilización orgánica, y la fertilización inorgánica y la fertilización con materia orgánica complementada con sulfato de potasio. La más baja producción se obtuvo sin fertilización. Martins y Furlani (2010) indican que es de esperar que plantaciones de café, que utilicen cultivares adaptados a condiciones ambientales regionales con una densidad de población óptima y una nutrición adecuada muestren respuestas de alto rendimiento.

En términos generales, las producciones obtenidas con las tres densidades de siembra estudiadas fueron relativamente bajas, comparadas con las registradas en estudios similares en esta localidad. Farfán y Mestre (2004) reportan producciones cercanas a los $11.263 \mathrm{~kg} \mathrm{ha}^{-1}$ de café pergamino seco, acumulado durante seis cosechas, con una densidad de siembra de 4.500 plantas/ha de café y bajo sombrío de Inga edulis. La respuesta del café a la fertilización dependerá del estado nutricional del suelo, la extensión de la sombra, la absorción de nutrientes por el árbol de sombra y el reciclaje de los nutrientes a través de la poda y la hojarasca (Jessy, 2011).

En la fertilización del café bajo sombrío pueden adoptarse alternativas de fertilización como la aplicación de materia orgánica en mezcla con fuentes de potasio como el $\mathrm{K}_{2} \mathrm{SO}_{4} .2 \mathrm{MgSO}_{4}$, comercializado como Sulpomag, siempre que se establezcan altas densidades de siembra. Aplicación de tecnologías donde se combinen los fertilizantes inorgánicos con los orgánicos son estrategias adecuadas para reducir los costos de producción, incrementar la productividad y realizar una fertilización equilibrada (Melke \& Ittana, 2015).

El café establecido a densidades inferiores a 6.000 plantas/ha, puede ser fertilizado con fuentes orgánicas o inorgánicas. Es recomendable realizar las evaluaciones del porcentaje de cobertura o de sombreamiento, hacer su regulación hasta obtener un porcentaje de acuerdo al número de horas de brillo solar al año (Farfán \& Jaramillo, 2009) y posteriormente realizar la fertilización, debido a que la respuesta del café a la fertilización está determinada por la disponibilidad de luz dentro del cultivo. Con el empleo de subproductos de la producción de café se obtienen beneficios adicionales como regulación del pH del suelo e incrementos del fósforo y potasio, aparte de los beneficios ecológicos asociados al cultivo (Haggar et al., 2011).

\section{LITERATURA CITADA}

Cuzato-Mancuso, M. A., Peres-Soratto, R., Costa, A. A., \& Amaral, G. S. (2014). Effect of potassium sources and rates on arabica coffee yield, nutrition, and macronutrient export. Revista Brasileira de Ciência do Solo, 38(5), 1448-1456. https://doi.org/10.1590/ S0100-06832014000500010

Farfán, F., \& Mestre, A. (2004). Fertilización del café en un sistema agroforestal en la zona cafetera norte de 
Colombia. Revista Cenicafé, 55(3), 232-245. http://hdl. handle.net/10778/255

Farfán, F., \& Jaramillo,A. (2009). Sombrío para el cultivo del café según la nubosidad de la región. Avances Técnicos Cenicafé, 379, 1-8. http://hdl.handle.net/10778/376

Federación Nacional de Cafeteros de Colombia. (2020, s. f.). Estadísticas cafeteras, Precios, área y producción de café. https://federaciondecafeteros.org/wp/estadisticascafeteras

González, H. (2013). Identificación de las principales Unidades de suelos de la zona cafetera. En Federación Nacional de Cafeteros de Colombia (Ed.), Manual del cafetero colombiano: Investigación y tecnología para la sostenibilidad de la caficultura (Vol. 1, pp. 269-283). Cenicafé.

Haggar, J., Barrios, M., Bolaños, M., Merlo, M., Moraga, P., Munguia, R., Ponce, A., Romero, S., Soto, G., Staver, C., \& Virginio, E. (2011). Coffee agroecosystem performance under full sun, shade, conventional and organic management regimes in Central America. Agroforestry Systems, 82, 285-300. https://doi. org/10.1007/s10457-011-9392-5

Jessy, M. D. (2011). Potassium management in plantation crops with special reference to tea, coffee and rubber. Karnataka Journal of Agricultural Sciences, 24(1), 67-74.

Martins, P. M., \& Furlani, J. E. (2010). Yield performance and leaf nutrient levels of coffee cultivars under different plant densities. Scientia Agricola, 67(6), 720-726.https:// doi.org/10.1590/S0103-90162010000600015

Melke, A., \& Ittana, F. (2015). Nutritional Requirement and Management of Arabica Coffee (Coffea arabica L.) in Ethiopia: National and Global Perspectives. American Journal of Experimental Agriculture, 5(5), 400-418. https://doi.org/10.9734/AJEA/2015/12510

Montilla, J.,Arcila, J.,Aristizábal, M., Montoya, E.C., Puerta, G. I., Oliveros, C. E., \& Cadena, G. (2008). Propiedades físicas y factores de conversión del café en el proceso de beneficio. Avances Técnicos Cenicafé, 370, 1-8. http:// biblioteca.cenicafe.org/handle/10778/358

Ochoa, M., Rivera, R., Bustamante, C., \& Rodríguez, M. I. (2000). La fertilización fosfórica del Coffea arabica L. en suelo ferrítico rojo oscuro. Parte I. Fertilización Mineral. Cultivos Tropicales, 21(1), 73-79.

Roa, G., Oliveros, C. E., Álvarez, J., Ramírez, C. A., Sanz, J. R., Dávila, M. T., Álvarez, J. R., Zambrano, D. A., Puerta, G. I., \& Rodríguez, N. (1999). Lombricultura con subproductos del café. En Roa, G., Oliveros, C.E., Álvarez, J., Ramírez, C.A., Sanz, J.R., Dávila, M.T., Álvarez, J.R., Zambrano, D.A., Puerta, G.I., \& Rodríguez, N (Eds.), Beneficio ecológico del café (pp. 202-209). Cenicafé.

Sadeghian., S. (2008). Fertilidad del suelo y nutrición del café en Colombia. Boletín Técnico Cenicafé, 32, 1-44. http://hdl.handle.net/10778/587 